\title{
Neuroimage
}

\section{Anti-Ma2-associated encephalitis}

DOI: https://doi.org/10.17981/10.17981/JACN.1.1.2020.14

\section{Maria Agustina Ruiz-Yanzi ${ }^{1}$ \& Catalina Bensi ${ }^{1}$}

${ }^{1}$ Neurology department, Fleni (Buenos Aires, Argentina)

Correspondence:

\section{María Agustina Ruiz-Yanzi}

Neurology department, Fleni (Buenos Aires, Argentina)

Email:

maruiz@fleni.org.ar
A 30 year old male with no relevant previous medical history, consulted in the Emergency Room with a 1-month history of a sleep disorder with daily somnolence, adding 3 days before consultation temporal and spatial disorientation. Family members also referred hyperphagia. The patient didn't present headache, fever, behavioral manifestations, or any other systemic or neurological signs or symptoms. He denied recent trips or vaccination.

Neurological examination was normal.

A contrast enhanced brain MRI was performed, revealing in T2/ FLAIR weighted images a diffuse hyperintense hypothalamic and interpeduncular lesion with homogeneous contrast enhancement (Figure 1, A-B). General laboratory tests were normal, with negative HIV and VDRL results and normal B12 and thyroid function values. A lumbar puncture was performed, showing mononuclear pleocytosis and high protein levels, with a negative citologic test. Full body CT scan showed no alterations, and testicular ecography revealed hypoechoic lesions and macrocalcifications suggestive of testicular cancer.

Testicular exeresis was performed, with anatomy pathology findings of seminoma. Paraneoplastic and autoimmune antibodies in CSF showed positive results for anti-Ma2 antibodies.

It was concluded that the patient had an Anti-Ma2-associated encephalitis.

Chemotherapy was started and the patient received five pulses of methylprednisolone $1 \mathrm{~g}$, maintaining long term treatment with high doses of prednisone. The patient had subtle clinical improvement.

3 months later, the patient consulted in the emergency room for anterograde amnesia. A new brain MRI was done, showing improvement of the hypothalamic lesion but with bilateral hippocampal hyperintensity without contrast enhancement (Figure 1, C-D). It was interpreted that the patient had now added an antiMa2-associated limbic encephalitis. A new full body CT scan was done, showing new retroperitoneal adenopathies despite chemotherapy. The patient received immunoglobulin $2 \mathrm{~g} / \mathrm{kg}$ and started a new oncologic treatment, with modest clinical improvement in the following months.

A new brain MRI was performed a year later, showing only slight hyperintensity in the right wall of the third ventricle in T2/FLAIR sequences and no contrast enhancement (Figure 1, E-F). 


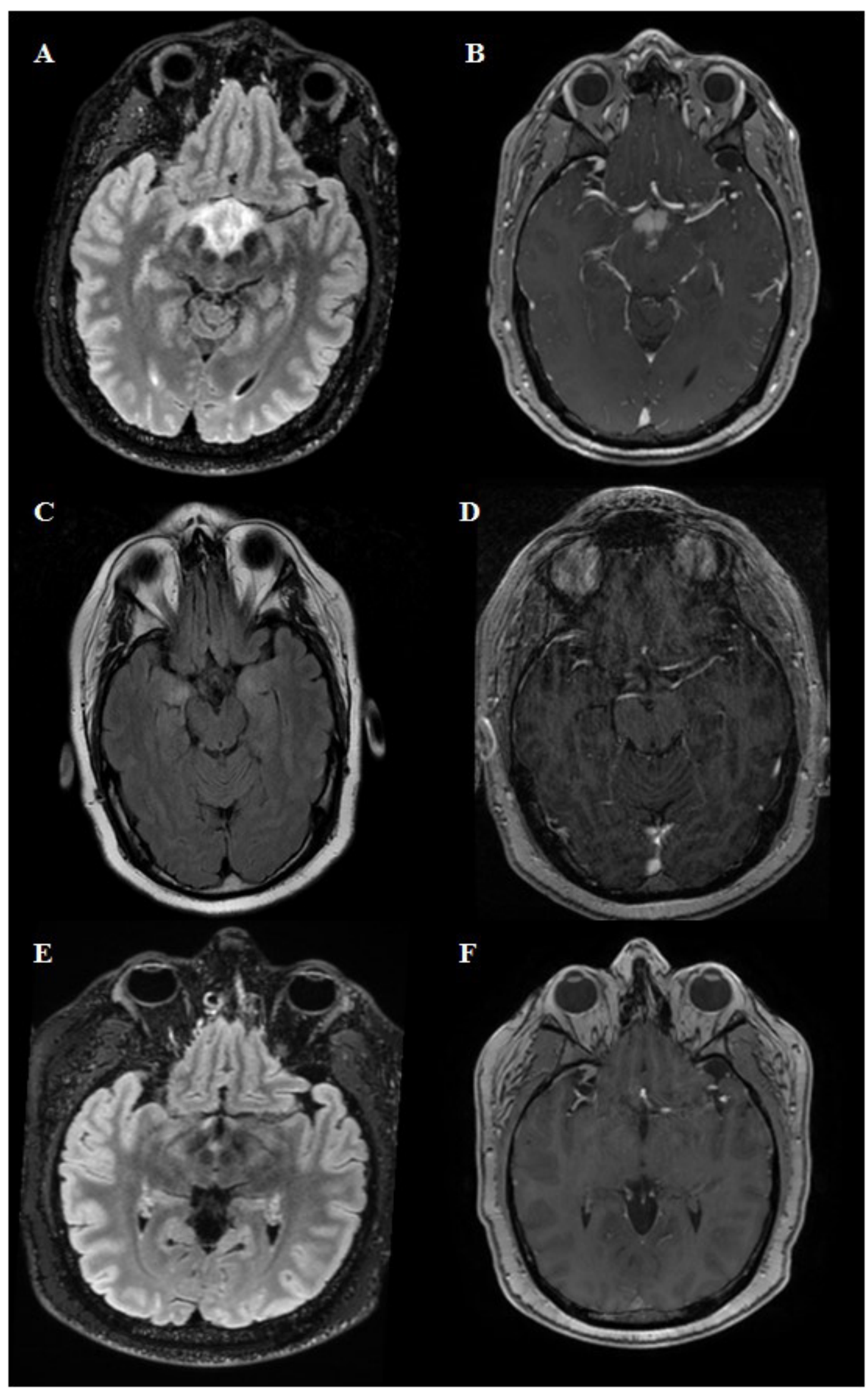

Figure 1. T2/FLAIR sequence (A) shows a diffuse hyperintense hypothalamic and interpeduncular lesion that is contrast enhancing (B). 3 months later, a new brain MRI was performed showing bilateral hippocampal hyperintensity (C) without contrast enhancement (D). Control brain MRI completed a year later shows remaining hyperintensity in the right wall of the third ventricle in T2/FLAIR sequence (E) and no contrast enhancement (F). Source: Author. 
Anti-Ma2 antibodies are usually present in men with testicular tumors, and they may be accompanied by a paraneoplastic syndrome including limbic, diencephalic or brainstem encephalitis (Ortega, Sola-Valls, Escudero, Saiz \& Graus, 2018). Brain MRI shows hyperintensity in the midbrain, diencephalon (thalamus/hypothalamus) or temporal lobes in T2/FLAIR weighted images, with almost half of these lesions presenting with contrast enhancement. CSF regularly has inflammatory changes (Ortega et al., 2018; Dalmau et al., 2004).

Clinical presentation usually varies according to the region affected, and these patients tend to have a good response to oncologic and immunologic treatment, with stabilization or even clinical improvement (Hoffmann et al., 2008).

Even though this is a rare entity, it is important to consider it as a differential diagnosis in cases of young men with midbrain or diencephalic lesions, since its early diagnosis and treatment could hinder its progression.

\section{REFERENCES}

Dalmau, J., Graus, F., Villarejo, A., Posner, J., Blumenthal, D., Thiessen, B., Saiz, A., Meneses, P. \& Rosenfeld, M. (2004). Clinical analysis of anti-Ma2-associated encephalitis. Brain, 127(8), 1831-1844. https://doi.org/10.1093/brain/awh203

Hoffmann, L., Jairus, S., Pellkofer, H., Schueller, M., Krumbholz, M., Koenig, F., Johannis, W., Fougere, C., Newman, T., Vicent, A. \& Voltz, R. (2008). Anti-Ma and anti-Ta associated paraneoplastic neurological syndromes: 22 newly diagnosed patients and review of previous cases. Journal of Neurology, Neurosurgery \& Psychiatry, 79(7), 767-773. https://doi.org/10.1136/ jnnp.2008.144170

Ortega, G., Sola-Valls, N., Escudero, D., Saiz, A. \& Graus, F. (2018). Anti-Ma and anti-Ma2-associated paraneoplastic neurological syndromes. Neurología (English Edition), 33(1), 18-27. https:// doi.org/10.1016/j.nrleng.2016.05.004

Maria Agustina Ruiz Yanzi: Fleni Neurology Resident (Buenos Aires, Argentina).

Catalina Bensi: Medical (University of Buenos Aires, Argentina). 